\title{
8. Zusammenarbeit mit den Ländern Osteuropas und der GUS
}

\section{Monika Egger}

\section{(2) OpenEdition \\ 12 Journals}

\section{Electronic version}

URL: http://journals.openedition.org/sjep/908

DOI: 10.4000/sjep.908

ISSN: 1663-9677

\section{Publisher}

Institut de hautes études internationales et du développement

\section{Printed version}

Date of publication: 1 mai 2002

Number of pages: 293-299

ISSN: $1660-5926$

\section{Electronic reference}

Monika Egger, « 8. Zusammenarbeit mit den Ländern Osteuropas und der GUS », Schweizerisches

Jahrbuch für Entwicklungspolitik [Online], 21 | 2002, Online erschienen am: 12 September 2012, abgerufen am 08 September 2020. URL : http://journals.openedition.org/sjep/908 ; DOI : https:// doi.org/10.4000/sjep.908 


\section{ZUSAMMENARBEIT MIT DEN LÄNDERN OSTEUROPAS UND DER GUS*}

I

M BERICHTSJAHR kann die Schweiz auf zehn Jahre Zusammenarbeit mit den Ländern Osteuropas blicken. Diese war geprägt durch eine grosse Dynamik, durch weitgehende Unvorhersehbarkeit der Ereignisse und auch durch Rückschritte aufgrund von Krisen und Kriegen in einzelnen Regionen. Die Transformation verläuft in den einzelnen Ländern und Regionen sehr unterschiedlich. In Zukunft will die Schweiz ihr Engagement weiter ausbauen und beantragt eine Aufstockung der Mittel.

Die Ostzusammenarbeit wird von der Abteilung für die Zusammenarbeit mit den Ländern Osteuropas und der GUS (AZO) der DEZA und dem Leistungsbereich Entwicklung und Transition des Staatssekretariats für Wirtschaft (seco) gemeinsam geplant und durchgeführt. Die DEZA ist zuständig für die technische Zusammenarbeit und das seco für die wirtschaftlichen und finanziellen Massnahmen. Die beiden Massnahmenbereiche lassen sich nicht immer scharf trennen; die verschiedenen Instrumente werden komplementär eingesetzt. Ziel ist eine möglichst auf die Bedürfnisse der Partnerländer abgestellte Zusammenarbeit. Seit 1999 bilden gemeinsam erarbeitete Landesprogramme die Grundlage für die Planung und Umsetzung. Die DEZA ist zuständig für die Gesamtkoordination.

\subsection{ZEHN JAHRE OSTZUSAMMENARBEIT}

Die Schweiz arbeitet seit 1990 mit den Staaten Osteuropas und seit 1992 auch mit Ländern der Gemeinschaft Unabhängiger Staaten (GUS) im Rahmen der Osthilfe zusammen. Der Bundesbeschluss über die Zusammenarbeit mit den Staaten Osteuropas von $1995^{1}$ bildet die aktuelle rechtliche Grundlage und hält als Zielsetzung der Osthilfe zwei zentrale Bereiche fest:

」 Förderung und Stärkung der Rechtsstaatlichkeit und der Menschenrechte sowie des Aufbaus und der Festigung des demokratischen Systems, namentlich stabiler politischer Institutionen.

- Die Förderung einer nachhaltigen wirtschaftlichen und sozialen Entwicklung.

Den ersten Rahmenkredit bewilligte das Parlament 1990. Nachfolgende Zusammenstellung zeigt die vom Parlament in Form von Rahmenkrediten und deren Aufstockungen insgesamt zur Verfügung gestellten Mittel für die Ostzusammenarbeit :

* Von Monika Egger, Ökonomin/Konsulentin im Bereich Wirtschaft und Entwicklung.

1. Bundesbeschluss vom 24. März 1995 über die Zusammenarbeit mit den Staaten Osteuropas. Amtliche Sammlung 1998/868. 
Tabelle 26: Mittel der Ostzusammenarbeit (in Mio. Franken)

\begin{tabular}{lcl}
\hline $\begin{array}{l}\text { Jahr } \\
\text { (Genehmigung durch das Parlament) }\end{array}$ & Betrag & Geografischer Geltungsbereich \\
\hline 1990: Rahmenkredit I & 900 & Polen, ehemalige CSSR, Ungarn \\
\hline 1991: Rahmenkredit II & 800 & zusätzlich Südosteuropa und die baltischen Staaten \\
\hline 1992: Aufstockung RK II & 600 & zusätzlich die Länder der neu gebildeten GUS \\
\hline 1998: Rahmenkredit III & 900 & Alle Länder \\
\hline 2002: Aufstockung RK III & 500 & Alle Länder \\
\hline Total & $\mathbf{3 7 0 0}$ & \\
\hline
\end{tabular}

Quelle: Daten AZO, eigene Zusammenstellung

Die bilaterale Zusammenarbeit der Schweiz mit den Oststaaten richtet sich - dies im Unterschied zur Entwicklungszusammenarbeit mit dem Süden - sowohl an die als Entwicklungsländer eingestuften Staaten ${ }^{2}$ wie auch an die anderen ausgewählten Länder und Regionen Osteuropas, die in ihrem Transformationsprozess auf zusätzliche Unterstützung angewiesen sind.

Aufgrund strategischer und sicherheitspolitischer Überlegungen aber auch aus Effizienzgründen konzentriert die Schweiz ihre Unterstützung vorwiegend auf die Länder Südosteuropas, die im Vergleich zur Grösse und Bevölkerung der Länder der GUS beispielsweise mehr Mittel erhalten. Die Schweiz unterstützt die politische Stabilisierung und fördert die wirtschaftliche Entwicklung in diesen Ländern, was auch den Migrationsdruck abschwächt.

Eine weitere Konzentration der Hilfe erfolgt zugunsten der Länder Zentralasiens, welche Mitglieder der von der Schweiz angeführten Stimmrechtsgruppen bei den Internationalen Finanzinstitutionen und bei der EBRD sind.

Insgesamt waren per Ende 2000 aus dem ersten, zweiten und dritten Rahmenkredit zusammen 2'185,7 Millionen Franken im Rahmen der Ostzusammenarbeit verpflichtet.

Nach Regionen und nach Instrumenten aufgeteilt ergibt sich folgendes Bild.

Tabelle 27: Gesamtverpflichtungen per Ende 2000 (in Mio. Franken)

\begin{tabular}{lcccr}
\hline & $\begin{array}{c}\text { Technische } \\
\text { Zusammenarbeit }\end{array}$ & $\begin{array}{c}\text { Finanzielle } \\
\text { Zusammenarbeit }\end{array}$ & Kreditgarantien & Total \\
\hline Mitteleuropa & 198.6 & 407.0 & 123.1 & $\mathbf{7 2 8 . 7}$ \\
\hline Südosteuropa & 282.9 & 386.4 & 65.8 & $\mathbf{7 3 5 . 1}$ \\
\hline GUS & 269.9 & 261.0 & 191.0 & $\mathbf{7 2 1 . 9}$ \\
\hline Total & $\mathbf{7 5 1 . 5}$ & $\mathbf{1 0 5 4 . 4}$ & $\mathbf{3 7 9 . 9}$ & $\mathbf{2 1 8 5 . 7}$ \\
\hline
\end{tabular}

Quelle: DEZA/seco, Jahresbericht 2000 der internationalen Zusammenarbeit der Schweiz, Broschüre "Zusammenarbeit mit Osteuropa».

Die technische Zusammenarbeit wird von der AZO/DEZA durchgeführt. Sie unterstützt den politischen und sozialen Transformationsprozess in den Partnerländern. Die Instrumente sind Beratungsleistungen (beispielsweise für besseren Zugang zu Krediten, Stärkung und Vernetzung von Institutionen, Mediation in Konfliktsituationen) und Lieferung von Ausrüstungsgütern.

2. Folgende Länder Mittel- und Osteuropas sowie der GUS sind als Entwicklungsländer eingestuft (OECD-Liste 1, öffentliche Entwicklungshilfe): Albanien, Aserbaidschan, Georgien, Kasachstan, Kirgistan, Moldawien, Slowenien, Tadschikistan, Turkmenistan, Usbekistan. 
Der Einsatz der Instrumente richtet sich nach den prioritären Aktionsbereichen, wie sie in der Strategie 2010 der DEZA sowohl für die Ostzusammenarbeit wie für die Entwicklungszusammenarbeit festgehalten sind:

- Krisenprävention und Krisenbewältigung: Sie umfasst den Politikdialog mit den Regierungsstellen und die Förderung und Unterstützung von Initiativen auf der Ebene der Institutionen und der Gemeinden, Rückkehrhilfeprogramme und auch Nothilfe und Wiederaufbau.

口 Gute Regierungsführung: Förderung einer verantwortungsvollen Verwaltungsführung und eines guten Zusammenwirkens von Staat und Zivilgesellschaft.

口 Einkommensförderung und Beschäftigung: Die Förderung des Zugangs zu Arbeit und Einkommen umfasst ein breites Massnahmenpaket, das einen Beitrag zur Umkehrung des negativen Trends des Nettoabbaus an Arbeitsplätzen in vielen Ländern Osteuropas leisten will. Dazu gehört auch der Kampf gegen Korruption.

- Förderung der sozialen Gerechtigkeit: Der Transformationsprozess führte für viele Menschen in den meisten Ländern zu einer Verschlechterung ihrer Lebensqualität und zu Unsicherheit über die Zukunftsperspektiven, besonders auch für die Jungen. Diese Entwicklung drückt sich aus in der Verarmung breiter Bevölkerungsschichten, der sog. «neuen Armut». Massnahmen in diesem Bereich setzen vor allem in der Verbesserung der Dienstleistungen in den Sektoren Arbeitsmarkt, Gesundheit und Bildung an. Sie umfasst auch die Massnahmen zur Integration für Rückkehrende.

- Nachhaltige Nutzung der natürlichen Ressourcen: Die nachhaltige Verbesserung der Umweltsituation setzt auf vielen Ebenen an. Sie beinhaltet beispielsweise auch die Ausarbeitung einer Umweltgesetzgebung und deren Inkraftsetzung. Die Schweiz setzt auf eine möglichst breite Einbindung aller Akteure: Regierung, Wirtschaft und Zivilgesellschaft.

Die finanzielle Zusammenarbeit fördert die Schaffung marktwirtschaftlicher Strukturen. Massnahmen sind die Verbesserung der Rahmenbedingungen, die Förderung privater Investitionen, Unterstützung und Bereitstellung der Basisinfrastruktur und die Förderung des Handels. Zuständig für diesen Bereich ist das seco.

- Handels- und Investitionsförderung: Ziel ist die Verbesserung der Handelspolitiken und der Investitionspolitiken der Partnerländer, die Förderung der wirtschaftlichen Integration und die Stärkung der Strukturen. Massnahmen können beispielsweise sein: die Schaffung von Berufsverbänden, die Strukturierung und Organisation des Finanzsektors, Beratung und Vermittlung im Bereich von Investitionsprojekten, Schaffung und Vermittlung von Risikokapitalfonds.

- Kreditgarantien: Kreditgarantien aus den Osteuropa-Rahmenkrediten kommen in jenen Ländern zum Tragen, in denen die schweizerische Exportrisikogarantie nicht oder nur teilweise verfügbar ist. Sie ermöglichen es diesen Ländern, Investitionsgüter zu importieren, die für ihre wirtschaftliche Entwicklung wichtig sind. Die schweizerischen Unternehmen können mit den Kreditgarantien die erhöhten Risiken für ihre Lieferungen decken. Die 
verpflichteten Kreditgarantien werden nur im Schadenfall ausgabenwirksam. Im Jahre 2000 beispielsweise war kein solcher Schadenfall eingetreten.

- Zahlungsbilanzhilfen und Entschuldungsmassnahmen: Durch den Transformationsprozess geraten Zahlungsbilanz und Staatsbudget stark unter Druck. Mit der Gewährung von Zahlungsbilanzhilfen und Budgethilfen und mit Entschuldungsmassnahmen unterstützt die Schweiz die Umsetzung wichtiger Reformschritte.

Finanzierungszuschüsse: Sie unterstützen konkrete Infrastrukturprojekte und bilden den volumenmässig stärksten Massnahmenbereich.

Nachfolgende Tabelle zeigt die Verwendung der Mittel nach Instrumenten ${ }^{3}$.

Tabelle 28: Sektorielle Aufteilung der Osthilfe (in Mio. Franken)

\begin{tabular}{|c|c|c|}
\hline & Verpflichtungen & Auszahlungen $2000^{*}$ \\
\hline Technische Zusammenarbeit & 751.5 & 77.0 \\
\hline Politik und Staatsaufbau & 229.3 & \\
\hline Wirtschaft/Bildung & 117.6 & \\
\hline Wissenschaft/Kultur & 108.6 & \\
\hline Landwirtschaft & 104.5 & \\
\hline Gesundheit/Soziales & 104.2 & \\
\hline Energie/Umwelt & 87.1 & \\
\hline Finanzielle Zusammenarbeit & & 111.1 \\
\hline Finanzierungszuschüsse & 842.6 & 97.7 \\
\hline Umwelt & 287.3 & \\
\hline Energie & 178.6 & \\
\hline Gesundheitswesen & 122.6 & \\
\hline Infrastruktur (Kataster, Metrologie) & 60.7 & \\
\hline Bankwesen & 48.0 & \\
\hline Telekommunikation & 43.7 & \\
\hline Transport & 21.5 & \\
\hline Verarbeitete Landwirtschaftsprodukte & 13.8 & \\
\hline Diverse Bereiche & 66.2 & \\
\hline Kreditgarantien** & 379.0 & - \\
\hline Zahlungsbilanzhilfen/Entschuldungsmassnahmen & 23.2 & - \\
\hline Handels- und Investitionsförderung & 143.8 & 13.4 \\
\hline Total & 2140.9 & 188.2 \\
\hline
\end{tabular}

Quelle: DEZA/seco, Jahresbericht 2000 der internationalen Zusammenarbeit der Schweiz, Broschüre "Zusammenarbeit mit Osteuropa».

* Das Detail der Auszahlungen 2000 ist nicht verfügbar (Stand Januar 2002).

${ }^{* *}$ Kreditgarantien werden nur im Schadenfall ausgabenwirksam.

\subsection{AUFWENDUNGEN 2000}

Im Jahre 2000 wurden insgesamt 188,2 Millionen Franken aufgewendet; davon 77 Millionen Franken für Massnahmen der technischen Zusammenarbeit und 111,1 Millionen Franken für die finanzielle Zusammenarbeit.

Mehr als die Hälfte der Auszahlungen flossen in die Region Südosteuropa, die $49 \%$ der technischen und $60 \%$ der finanziellen Zusammenarbeit absorbierte.

3. Die in diesem Kapitel angegebenen Zahlen können von den Zahlen im Statistischen Teil des Jahrbuchs leicht abweichen, da letzere im Februar 2002 aktualisiert wurden. 
Tabelle 29: Auszahlungen 2000 nach Regionen (in Mio. Franken)

\begin{tabular}{lcc}
\hline & Technische Zusammenarbeit & Finanzielle Zusammenarbeit \\
\hline Mitteleuropa & 1.4 & 20.6 \\
\hline Südosteuropa & 38.1 & 70.7 \\
\hline GUS & 28.0 & 19.5 \\
\hline Regional nicht aufgeteilt & 9.4 & 6.3 \\
\hline Total & $\mathbf{7 6 . 9}$ & $\mathbf{1 1 7 . 1}$ \\
\hline
\end{tabular}

Quelle: DEZA/seco, Jahresbericht 2000 der internationalen Zusammenarbeit der Schweiz, Broschüre "Zusammenarbeit mit Osteuropa».

\subsection{AUFSTOCKUNG UND VERLÄNGERUNG DES RAHMENKREDITS III}

Aufgrund der Unvorhersehbarkeit der Ereignisse und der gesteigerten Bedürfnisse in den Partnerländern wurden in den letzten Jahren mehr Mittel aus dem Rahmenkredit III beansprucht, als bei der Planung vorgesehen war, namentlich in den Ländern Südosteuropas, speziell im Kosovo. Die Gründe waren :

口 Der Kosovo-Krieg im Jahre 1999 und dessen Folgen in der Region.

- Stabilitätspakt Südosteuropa: Unter dem Eindruck des Kosovo-Krieges wurde Mitte 1999 der «Stabilitätspakt für Südosteuropa» lanciert mit dem Ziel, langfristig Stabilität und nachhaltige Entwicklung in den Ländern Südosteuropas zu fördern. Die Mitgliedsländer des Paktes, dem die Schweiz im Juni 2000 beigetreten ist, verpflichten sich zu zusätzlichen finanziellen Engagements. Die Partnerländer ihrerseits engagieren sich für umfassende Reformen und eine verbesserte interregionale Zusammenarbeit.

- Die Bundesrepublik Jugoslawien (BRJ) wurde nach den Wahlen im Herbst 2000 zu einem Schwerpunktland der schweizerischen Ostzusammenarbeit. Die Schweiz unterstützt die Reformbemühungen seither mit einem Sonderprogramm.

口 Der Reformprozess in den Ländern Zentralasiens und des Kaukasus verläuft langsamer und schwieriger und beansprucht mehr Mittel als ursprünglich angenommen.

Aufgrund dieser Erfahrungen will sich die Schweiz zu einem verstärkten Engagement in der Ostzusammenarbeit verpflichten, das auch im «Aussenpolitischen Bericht 2000 » vorgesehen ist. Die wichtigsten Gründe für den Ausbau sind:

- Wiederaufbau, Entwicklung und Stabilisierung in der Region Südosteuropa.

口 Verstärkung der Schweizer Unterstützung der Länder ihrer Stimmrechtsgruppen in Zentralasien und im Kaukausus ${ }^{4}$.

口 Die Unvorhersehbarkeit der Entwicklung aufgrund der unstabilen Lage und der Dynamik der Transformation erfordert genügend Mittel für die notwendigen Anpassungen an die jeweils neuen Gegebenheiten.

4. IWF/Weltbankgruppe: Aserbeidschan, BRJ, Kirgistan, Polen, Schweiz, Tadschikistan, Turkmenistan, Usbekistan.

EBRD-Gruppe: Aserbeidschan, BRJ, Kirgistan, Liechtenstein, Schweiz, Türkei, Turkmenistan, Usbekistan. 
- Generell verpflichtet sich die Schweiz zu mehr Mitteln für die Entwicklungszusammenarbeit, um die $0.4 \%$ des BSP bis ins Jahr 2010 zu verwirklichen. Davon sollen auch die Entwicklungsländer Osteuropas profitieren.

Deshalb beantragt der Bundesrat dem Parlament die Aufstockung des dritten Rahmenkredites um weitere 500 Millionen Franken, mit gleichzeitiger Verlängerung seiner Geltungsdauer um weitere 2 Jahre bis 2005. Zu diesem Zeitpunkt steht die Erneuerung des Bundesbeschlusses an. Der neue Bundesbeschluss könnte zusammen mit der Botschaft zum vierten Rahmenkredit erarbeitet werden, begründet der Bundesrat seine strategische Planung der Aufstockung.

Grundsätzlich will die Schweiz ihre Osthilfe bis 2005 ausbauen und noch vermehrt auf die Regionen und Bevölkerungsgruppen ausrichten, welche durch die Transformation besonders nachteilig betroffen sind. Neue Armut und die anhaltende Verarmung breiter Bevölkerungskreise, mangelnde soziale Unterstützung und fehlende wirtschaftliche Perspektiven bergen das Risiko von Resignation und Konflikten. Aus heutiger Sicht müsse von einem grossen und langfristigen Engagement zur Unterstïtzung der Transformation in diesen Ländern und Regionen ausgegangen werden. Mit ihrem Engagement in der Armutsbekämpfung in Osteuropa leiste die Schweiz ihren Beitrag an die Verwirklichung der internationalen Entwicklungsziele, welche aus den Weltkonferenzen der 90er Jahre unter der Ägide der UNO hervorgegangen sind (UN-Milleniums-Deklaration) 5 .

Die geografischen Schwerpunkte werden beibehalten, d.h. dass Südosteuropa die stärkste Unterstützung erfährt. Die Zusammenarbeit mit den Ländern Zentralasiens wird ausgebaut; Kirgistan bleibt Schwerpunktland der Zusammenarbeit.

\section{$\square$ Entwicklung in der Verwaltung}

Die beratende Kommission des Bundesrates für internationale Entwicklungszusammenarbeit wurde um die «Subkommission Ostzusammenarbeit» erweitert.

Auf der strategischen Ebene wurden in der Verwaltung einige organisatorische Massnahmen eingeleitet, und die Zusammenarbeit zwischen der DEZA und dem seco ist geklärt und verstärkt worden. Ein gemeinsames «Strategiekomitee DEZA/seco» legt die strategische Ausrichtung der Ostzusammenarbeit fest. Ein «Komitee für Einsatzpolitik Ostzusammenarbeit» koordiniert auf der Stufe der Abteilungen den Einsatz der Mittel und beschliesst über die Errichtung oder die Schliessung von gemeinsamen Koordinationsbüros. Solche Büros gibt es in Tirana, Sarajewo, Sofia, Skopje, Bukarest, Belgrad, Pristina, Moskau, Kiew, Tbilisi, Duschanbe, Biskek, Taschkent (Subbüro). In Moldawien und in Belarus führt die Abteilung Humanitäre Hilfe ein Koordinationsbüro.

5. Zusatzbotschaft über die Aufstockung und Verlängerung des Rahmenkredits III für die Zusammenarbeit mit Osteuropa. 


\section{QUELLEN}

\section{Dokumente}

DEZA/seco, Jahresbericht 2000 der internationalen Zusammenarbeit der Schweiz, Broschüre «Zusammenarbeit mit Osteuropa».

DEZA/seco, Zusatzbotschaft über die Aufstockung und Verlängerung des Rahmenkredits III für die Zusammenarbeit mit Osteuropa.

NZZ, 15.11.2001 (Mehr Geld für humanitäre Hilfe und Osteuropa)

La Vie économique - Revue de politique économique 11/2000: La Task Force «Europe du Sud-Est» au Secrétariat d'Etat à l'économie.

\section{INTERNET-ADRESSEN}

DEZA: <www.deza.admin.ch>.

seco: <www.seco.admin.ch>. 